\title{
CircCSNKIGI Contributes to the Tumorigenesis of Gastric Cancer by Sponging miR-758 and Regulating ZNF2I7 Expression
}

\section{Feng Qiang \\ Jingjing $\mathrm{Li}$}

Department of Gastroenterology, the First People's Hospital of Huzhou, Huzhou, 313000, People's Republic of China
Correspondence: Jingjing Li Department of Gastroenterology, the First People's Hospital of Huzhou, No. I58, Guangchanghou Road, Huzhou, Zhejiang, 313000, People's Republic of China

Email li_jingjing I @@I63.com
Background: Increasing evidence indicates that circular RNAs (circRNAs) act as vital regulators in various cancers. Nevertheless, the effect of circCSNK1G1 on gastric cancer (GC) is still unknown.

Methods: The mRNA levels of circCSNK1G1, miR-758, and ZNF217 were measured by RT-qPCR. The protein levels of ZNF217 were evaluated by Western blotting. Cell migration, invasion, proliferation, and apoptosis were detected by Transwell, CCK-8, and flow cytometry assays. The association between miR-758 and circCSNK1G1/ZNF217 was confirmed by RIP and luciferase reporter assays. Xenograft assay was employed for in vivo experiment. Results: In the current study, it was demonstrated that the expression levels of circCSNK1G1 and ZNF217 were upregulated in GC tissues and cells, while the level of miR-758 was declined. Furthermore, functional assays indicated that circCSNK1G1 depletion suppressed GC progression in vitro and in vivo. In addition, circCSNK1G1 directly interacted with miR-758, and the supplementation of miR-758 suppressed the development of $\mathrm{GC}$, which was abolished following pcDNA3.1-circCSNK1G1 transfection. Then, we explored the downstream mechanism of miR-758 and found that miR-758 could target the 3' UTR of ZNF217 mRNA. The overexpression of miR-758 neutralized the ZNF217-mediated effects on facilitating the progression of GC. Finally, we revealed that circCSNK1G1 could upregulate ZNF217 expression by sponging miR-758 in GC cells.

Conclusion: Our study revealed that circCSNK1G1 accelerated GC progression via the miR-758/ZNF217 axis, suggesting that circCSNK1G1 might be a potential biomarker for GC diagnosis and treatment.

Keywords: gastric cancer, GC, circCSNK1G1, miR-758, ZNF217

\section{Introduction}

Gastric cancer (GC) is the third most frequent malignancy in the world ${ }^{1}$ and nearly a million new cases of GC are reported annually, with two-thirds of all cases in developing countries. ${ }^{2,3}$ Despite great progress has been achieved in the diagnosis and therapy of $\mathrm{GC}$, the five-year survival rate remains unsatisfactory owing to the high metastasis and recurrence of GC. ${ }^{4,5}$ Therefore, the identification of novel possible biomarkers for GC diagnosis and treatment is critical.

Circular RNAs (circRNAs) are a category of RNA with a covalent closed loop structure. ${ }^{6-8}$ Increasing research has indicated that circRNAs are involved in the development of various cancers through the regulation of cellular processes, such as cell growth, migration, apoptosis, and cell cycle. ${ }^{9}$ Liu et al disclosed that the 
upregulation of circRNA-5692 attenuated hepatocellular carcinoma cell proliferation, migration, and invasion in vitro and inhibited tumor growth in vivo. ${ }^{10}$ Zeng et al revealed that c-Myb-induced circHIPK3 facilitated cell growth and metastasis by regulating miR-7 expression in colorectal cancer. ${ }^{11}$ Moreover, many circRNAs have been identified as important regulators in GC. To cite an instance, $\mathrm{Yu}$ et al identified down-regulated expression and vital diagnostic value of circ_0067582 in GC. ${ }^{12}$ A report from Sun et al uncovered that circMAN2B2 facilitated GC cell proliferation and metastasis by acting as a sponge for miR-145. ${ }^{13}$ It was also revealed that circ_0023642 contributed to GC development via interaction with miRNA-223. ${ }^{14}$ Ding et al reported that circCSNK1G1 was one of the markedly upregulated circRNAs in GC. ${ }^{15}$ However, the biological role of circCSNK1G1 in GC is still unclear.

MicroRNAs (miRNAs), another type of ncRNAs, frequently participate in the regulation of the tumorigenesis of multiple human cancers, including GC. ${ }^{16}$ For example, the supplementation of miR-129 suppressed the tumorigenesis of GC by targeting COL1A1. ${ }^{17}$ MiR-4317 impeded GC cell proliferation by inhibiting ZNF322. ${ }^{18}$ miR-197 repressed GC cell viability and invasion through mediating MTDH. ${ }^{19}$ The dysregulation of miR-758 has been reported to participate in the development of various human cancers, including bladder, ${ }^{20}$ ovarian, ${ }^{21}$ and cervical cancers. ${ }^{22}$ Moreover, Guo et al identified that miR-758 played an antitumor role in the GC progression. ${ }^{23}$ Nonetheless, the exact function and molecular mechanism of miR-758 in GC need to be further explained.

In this research, we uncovered a new mechanism that circCSNK1G1 modulated the miR-758/ZNF217 axis to facilitate GC progression. Our findings suggest that circCSNK1G1 may be a novel biomarker for GC diagnosis and treatment.

\section{Materials and Methods Clinical Samples}

From the First People's Hospital of Huzhou, 48 GC tissues and 48 corresponding normal tissues were collected. Besides, serum samples were also collected from 48 healthy volunteers as a healthy control grouThis study was conducted in accordance with the Declaration of Helsinki and approved by the Ethics Committee of the First People's Hospital of Huzhou, and each participant provided the informed consent. None of the patients received preoperative treatment or had been diagnosed with other cancers. The specimens were quickly frozen in liquid nitrogen and stored at $-80^{\circ} \mathrm{C}$ for further study.

\section{Cell Line Culture}

Human GC cell lines (AGS, HGC-27, and MKN45) and normal gastric epithelial cell line (GES-1) were purchased from ATCC. Cells were incubated in RPMI 1640 (HyClone, UT, USA) containing 10\% FBS (Gibco, Rockville, MD, USA) maintained in a $37^{\circ} \mathrm{C}, 5 \% \mathrm{CO}_{2}$ incubator.

\section{Cell Transfection}

siRNA targeting circCSNK1G1 (si-circCSNK1G1\#1: 5'GGUACCGACGACUAGUCUUGU-3'; si-circCSNK1G 1\#2: 5'- UGGCCCUCGUAACCCAGCUGAU-3') with its control group (si-NC), miR-758 mimics (5'-UUUGU GACCUGGUCCACUAACC-3') with its control (NC mimics, 5'-ACAUCUGCGUAAGAUUCGAGUCUA-3'), miR-758 inhibitor (5'-GGUUAGUGGACCAGG UCACAAA-3') with its control (NC inhibitor, 5'GCGUAACUAAUACAUCGGAUUCGU-3') were obtained from GenePharma (Shanghai, China). circCSNK1G1 or ZNF217 overexpression plasmid (pcDNA3.1-circCSNK1G1 or pcDNA3.1-ZNF217) was established by inserting the fulllength circCSNK1G1 or ZNF217 sequence into pcDNA3.1 vector. Lipofectamine 2000 (Invitrogen, CA, USA) was performed to transfect these oligonucleotides or vectors into GC cells.

\section{RT-qPCR}

Trizol reagent (Invitrogen) was used for the extraction of RNA. cDNA was then synthesized using PrimeScript RT Reagent kit (Takara, Otsu, Japan) qPCR was performed using the SYBRGreen PCR kit (Takara) and a 7500 Fast PCR system (Applied Biosystems; Thermo Fisher Scientific, Inc.). GAPDH or U6 was used as reference control, and the relative gene expression was calculated by the $2^{-\Delta \Delta \mathrm{Cq}}$ method. The primer sequences used were as follows: circCSNK1G1 forward, 5'-GCCATC ACAACAGCAGCCT-3' and reverse, 5'AGGTCAAACAAGTCCTCCAAG-3'; miR-758 forward, 5'-ACACTCCAGCTGGGTTTGTGACCTGGTC CA-3' and reverse, 5'-CTCAACTGGTGTCGTGGA GTCGGCAATTCAGTTGAGGGTTAGTG-3'; ZNF217 forward, 5'-GAGAAGCGAATGGTGAAAGC-3', and reverse, 5'-CAGCGCTCAAGTATGCAAAA-3'; GAPDH forward, 5'-GCACCGTCAAGGCTGAGAAC-3' and 
reverse, 5'-TGGTGAAGACGCCAGTGGA-3'; and U6 forward, 5'-CTCGCTTCGGCAGCACA-3' and reverse, 5'-AACGCTTCACGAATTTGCGT-3'

\section{Transwell Assay}

The abilities of invasion and migration of GC cells were detected using transwell chambers (Corning, NY, USA). For detecting invasion ability, the transwell chambers were coated with Matrigel (BD Biosciences). The transfected cells $\left(1 \times 10^{5}\right.$ cells) were suspended in RPMI 1640 without serum and added to the upper chambers. Then, the lower chamber was filled with $600 \mu \mathrm{L}$ RPMI 1640 supplemented with $10 \%$ FBS. After $24 \mathrm{~h}$, the invaded cells were stained with $0.5 \%$ crystal violet. The procedure for cell migration was the same as for cell invasion, except that the transwell chambers were not coated with Matrigel.

\section{CCK-8 Assay}

Transfected cells were seeded onto 96-well plates and then incubated for $0,24,48$, and $72 \mathrm{~h}$. The CCK-8 reagent was added to each well and then incubated for $4 \mathrm{~h}$. The absorbance value of each well was detected at $490 \mathrm{~nm}$.

\section{Xenograft Assay in Nude Mice}

5-week-old BALB/c nude mice bought from Shanghai Laboratory Animal Center (Shanghai, China) were used for the experiment. $1 \times 10^{7}$ AGS transfected with sicircCSNK1G1\#1 or si-NC were subcutaneously injected into mice. Every 7 days, the tumor volume was monitored. 28 days after injection, mice were sacrificed and tumors were weighted. The animal experiment was performed according to the recommendations in the Guide for the Care and Use of Laboratory Animals of the National Institutes of Health and approved by the Ethics Committee of the First People's Hospital of Huzhou.

\section{Immunohistochemistry (IHC)}

Tumor tissues collected from mice were processed by paraformaldehyde (4\%) for fixation, and then the fixed tissues were dehydrated and embedded in paraffin. Subsequently, sections (4-mm-thick) were obtained via intersecting the paraffin-embedded tissues. Then, sections were deparaffinized and incubated at $4{ }^{\circ} \mathrm{C}$ overnight with Ki67 antibody (Abcam). After incubation with a secondary antibody, sections were stained by hematoxylin, and photographed under a microscope.
RIP

RIP assay was applied using EZ-Magna RiP Kit (Millipore). Cells were lysed and incubated by anti-Ago2 antibody, with magnetic beads (Millipore, USA) or control antibody (Millipore, USA). The RT-qPCR was conducted after the immune precipitated RNA was purified.

\section{Flow Cytometry}

The transfected cells were digested with $0.25 \%$ trypsin, and then suspended in $100 \mu \mathrm{L}$ binding buffer to produce $1 \times 10^{6}$ cells/mL suspension. Subsequently, the suspension was added with $10 \mu \mathrm{L}$ AnnexinV-FITC and $10 \mu \mathrm{L}$ PI for $10 \mathrm{~min}$ in darkness, and the apoptosis of cells was analyzed using FACScan flow cytometer (BD Biosciences).

\section{Luciferase Reporter Assay}

Wild-type circCSNK1G1 (circCSNK1G1-WT), mutanttype (circCSNK1G1-Mut), wild-type ZNF217 (ZNF217WT), and mutant-type ZNF217 (ZNF217-Mut) were constructed by GenePharma. The reporter plasmids were cotransfection with miR-758 mimics or NC mimics via Lipofectamine 2000. The relative luciferase activity was detected by a dual-luciferase reporter assay system (Promega Corporation).

\section{Western Blotting}

Total protein was isolated from GC cells by using RIPA lysis buffer. The protein was separated with $10 \%$ SDS-PAGE and then moved to the PVDF membrane. After being blocked with 5\% skimmed milk, the membrane was mixed with primary antibodies (anti-ZNF217: ab136678 and antiGAPDH: ab181602; Abcam, USA) at $4^{\circ} \mathrm{C}$ all night. Then, the membrane was cultured with horseradish peroxidaselabeled secondary antibodies. Subsequently, the bands were evaluated with the enhanced chemiluminescence (ECL) Kit (Pierce, Thermo Fisher Scientific, IL, USA).

\section{Statistical Analysis}

The experiments were all conducted 3 times. Data were presented as mean \pm standard deviation. SPSS 17.0 was performed for statistical analysis. Student's $t$-test and ANOVA were utilized to measure the differences. Kaplan-Meier method was used to calculate the overall survival curve. $\mathrm{P}<0.05$ was considered as statistical significance. 


\section{Results}

CircCSNKIGI Was Upregulated in GC and Associated with the Poor Prognosis of GC Patients

Firstly, the levels of circCSNK1G1 in GC tissues and adjacent normal tissues were detected by RT-qPCR and the data showed that circCSNK1G1 was considerably upregulated in GC tissues (Figure 1A). In the clinical sample, Kaplan-Meier survival analysis showed that the GC patients with high expression of circCSNK1G1 exhibited a lower survival rate than those with low expression of circCSNK1G1 (Figure 1B). Besides, the upregulation of circCSNK1G1 was related to tumor size, histological grades and TNM stage, but not with patients' ages, gender or lymph node metastasis (Table 1). In addition, we also assessed the diagnostic value of serum circCSNK1G1, and the results showed that area under curve (AUC) was 0.8840 , implying that circCSNK1G1 might be used as an indicator for GC diagnosis (Figure 1C). Moreover, we further observed that GC cell lines (AGS, HGC-27, and MKN45) established higher circCSNK1G1 expression than GES-1 (Figure 1D). Hence, we speculated that circCSNK1G1 might be an oncogene of GC.

\section{Interference of circCSNKIGI Inhibited the Progression of GC in vitro}

To determine the function of circCSNK1G1 in GC, AGS and HGC-27 cells were transfected with sicircCSNK1G1\#1, si-circCSNK1G1\#2, or si-NC, and a remarkable downregulation of circCSNK1G1 level in cells transfected with si-circCSNK1G1\#1 and sicircCSNK1G1\#2 was detected by RT-qPCR (Figure 2A). CCK-8 assay revealed that circCSNK1G1 depletion
Table I The Relationship Between circCSNKIGI Expression Level and the Clinicopathologic Features of GC

\begin{tabular}{|c|c|c|c|c|}
\hline \multirow[t]{2}{*}{ Parameters } & \multirow[t]{2}{*}{ Number } & \multicolumn{2}{|c|}{ circCSNKIGI Expression } & \multirow[t]{2}{*}{ P-value } \\
\hline & & $\begin{array}{c}\text { Low } \\
\text { (<Median) }\end{array}$ & $\begin{array}{c}\text { High } \\
(\geq \text { Median) }\end{array}$ & \\
\hline \multicolumn{5}{|l|}{ Age (years) } \\
\hline$<65$ & 30 & 16 & 14 & 0.561 \\
\hline$\geq 65$ & 18 & 8 & 10 & \\
\hline \multicolumn{5}{|l|}{ Gender } \\
\hline Male & 26 & 12 & 14 & 0.75 \\
\hline Female & 22 & 12 & 10 & \\
\hline \multicolumn{5}{|l|}{ Tumor size } \\
\hline$<3 \mathrm{~cm}$ & 21 & 15 & 6 & 0.002 \\
\hline$\geq 3 \mathrm{~cm}$ & 27 & 9 & 18 & \\
\hline \multicolumn{5}{|l|}{ Histological grade } \\
\hline Well & 26 & 17 & 9 & 0.004 \\
\hline $\begin{array}{l}\text { Moderately and } \\
\text { poorly }\end{array}$ & 22 & 7 & 15 & \\
\hline \multicolumn{5}{|l|}{ TNM stage } \\
\hline $1+I I$ & 28 & 18 & 10 & 0.003 \\
\hline$I I I+I V$ & 20 & 6 & 14 & \\
\hline \multicolumn{5}{|l|}{$\begin{array}{l}\text { Lymph node } \\
\text { metastasis }\end{array}$} \\
\hline Absent & 36 & 20 & 16 & 0.158 \\
\hline Present & 12 & 4 & 8 & \\
\hline
\end{tabular}

restrained the proliferation of $\mathrm{GC}$ cells (Figure 2B). Furthermore, transwell assays indicated that the interference of circCSNK1G1 attenuated the migration and invasion of $\mathrm{GC}$ cells (Figure 2C and D). In addition, flow cytometry assay indicated that circCSNK1G1 knockdown promoted the cell apoptosis of GC (Figure 2E). These results demonstrated that the knockdown of circCSNK1G1 could inhibit GC development.
A

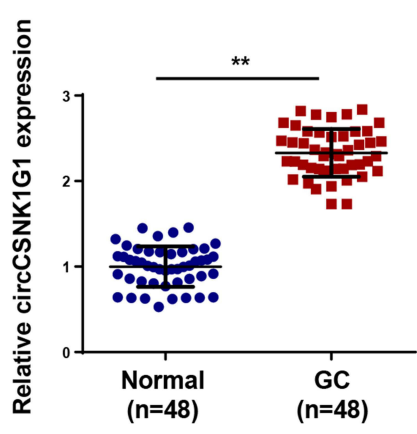

B

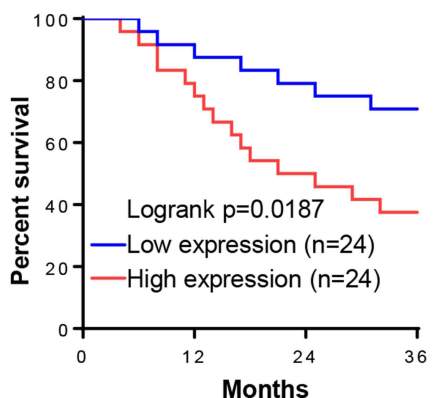

C

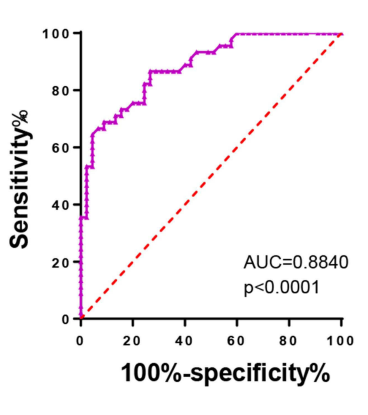

D

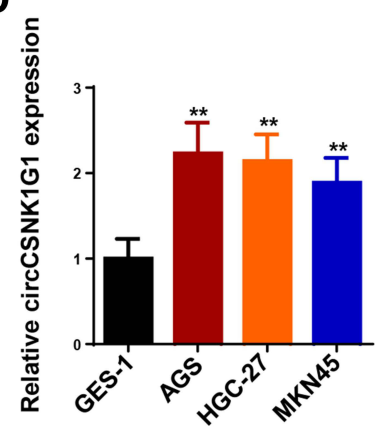

Figure I CircCSNKIGI was upregulated in GC and associated the prognosis of GC patients. (A) The level of circCSNKIGI in GC tissues and paired normal tissues was measured using RT-qPCR. (B) Kaplan-Meier analysis reveals the association of high crcCSNKIGI expression with poor overall survival in patients with GC. (C) ROC curve analysis was used to assess the diagnostic values of the circCSNKIGI. (D) The expression of circCSNKIGI in GC cell lines and normal gastric epithelial cell line was detected using RT-qPCR. **P $<0.01$. 
A

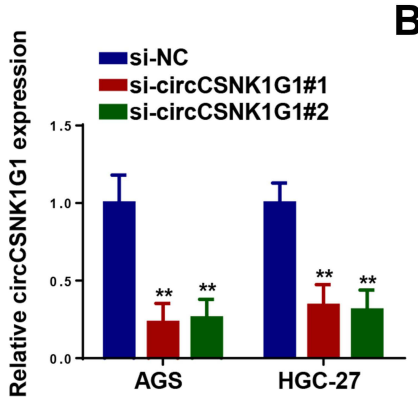

C

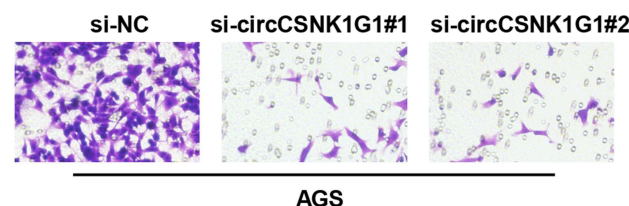

D

si-NC si-circCSNK1G1\#1 si-circCSNK1G1\#2

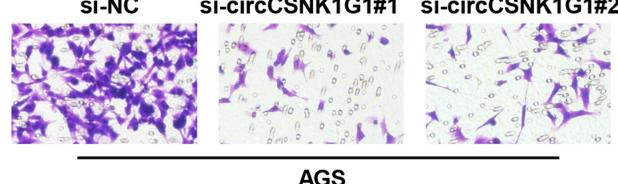

E

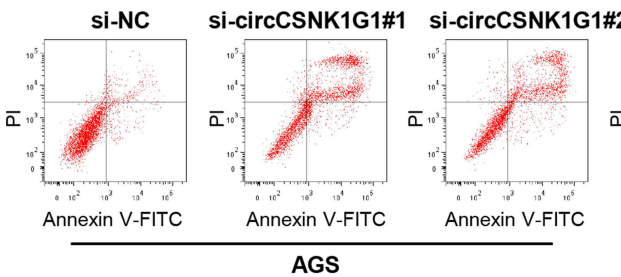

B

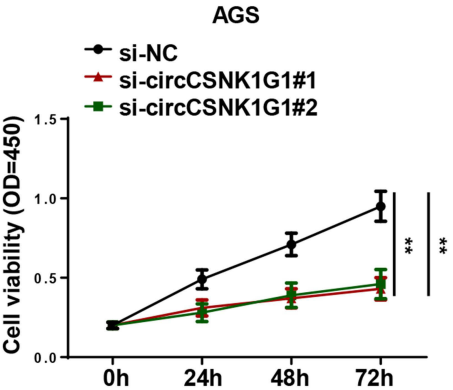

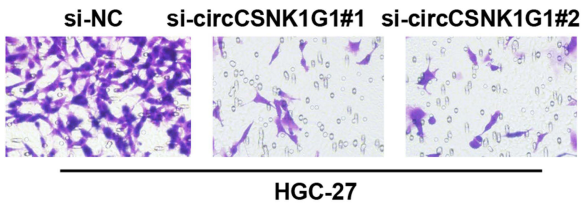

HGC-27
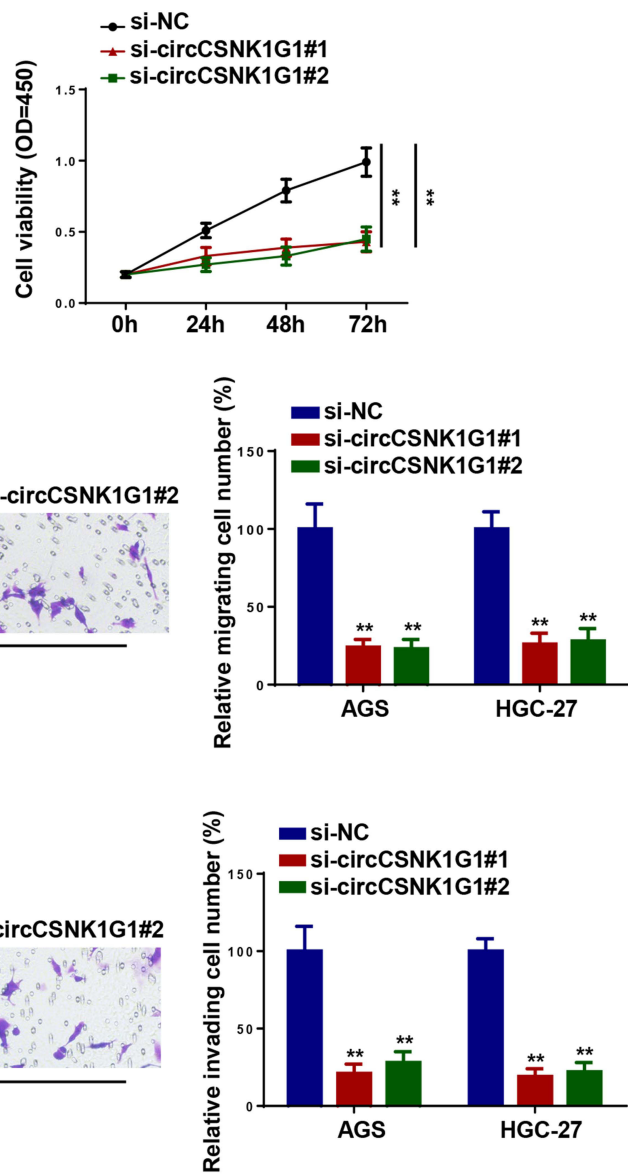

mi-NC

- si-circCSNK1G1\#1 - si-circCSNK1G1\#2
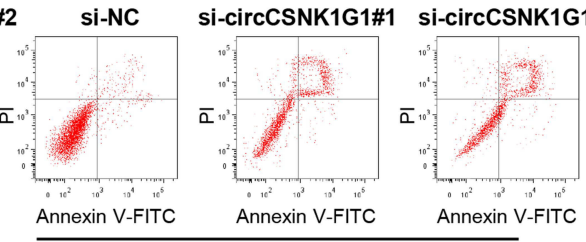

HGC-27

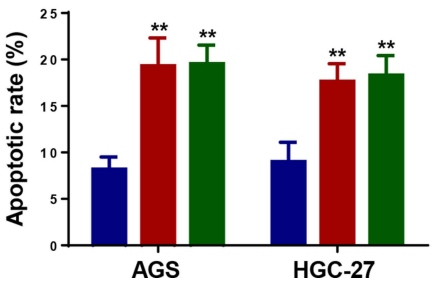

Figure 2 Interference of CircCSNKIGI inhibited the progression of GC. (A) The expression of circCSNKIGI was detected by RT-qPCR in AGS and HGC-27 cells transfected with si-circCSNKI GI\#I, si-circCSNKIGI\#2, or si-NC. (B) The proliferation of AGS and HGC-27 cells was measured by CCK-8 assay. (C and D) Transwell was used to detect the migration and invasion of AGS and HGC-27 cells transfected with si-circCSNKI GI\#I, si-circCSNKIGI\#2, or si-NC. (E) Flow cytometry assay showed the apoptosis of AGS and HGC-27 cells transfected with si-circCSNKIGI\#I, si-circCSNKIGI\#2, and si-NC. ${ }^{* * P}<0.01$.

\section{CircCSNKIGI Knockdown Represses GC Tumorigenesis in vivo}

To further elaborate the biological role of circCSNK1G1 in GC in vivo, AGS cells transfected with si-NC or sicircCSNK1G1\#1 were subcutaneously injected into nude mice. As presented in Figure 3A and B, circCSNK1 depletion significantly restrained tumor growth in mice. Moreover, IHC staining showed that circCSNK1 knockdown decreased Ki67 expression with comparison of the control group (Figure 3C). Altogether, these results implied that circCSNK1G1 knockdown suppressed GC tumorigenesis in vivo.

\section{CircCSNKIGI Was a Sponge of miR-758}

The downstream target genes were screened via the StarBase website to further explore the molecular mechanism of circCSNK1G1 in GC, and the results indicated that miR-758 might be a candidate downstream target of 

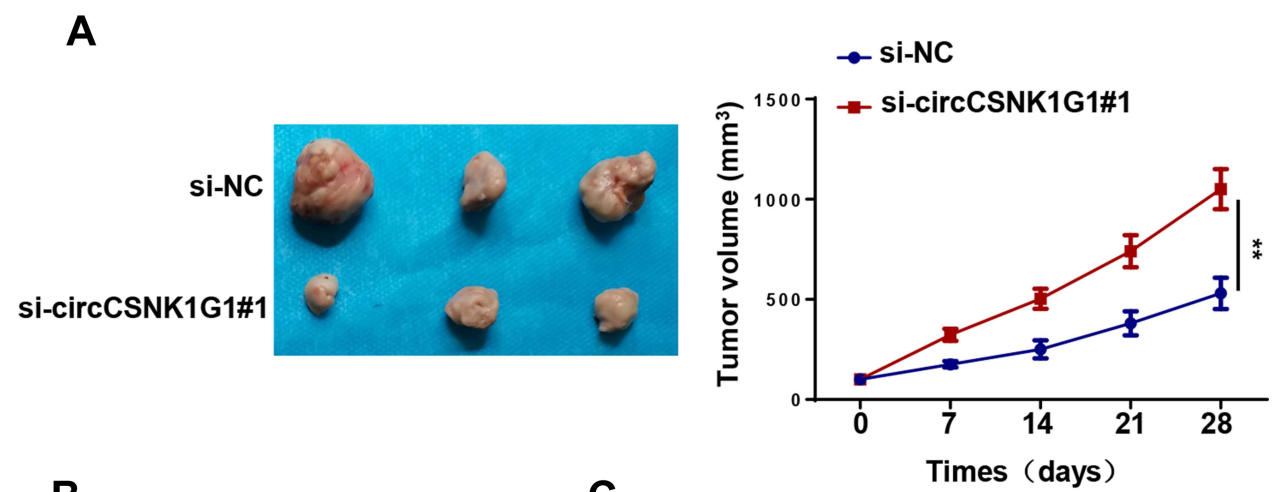

B

C
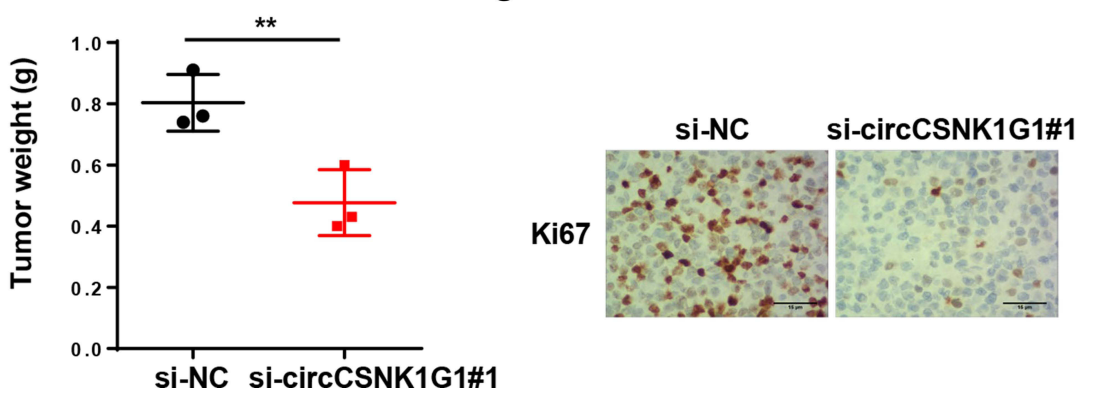

Figure 3 CircCSNKIGI knockdown represses GC tumorigenesis in vivo. (A) AGS cells stably transfected with si-circCSNKIGI\#I or si-NC were subcutaneously injected into nude mice. Tumor growth curves indicated that circCSNKIGI knockdown led to suppression on GC growth in mice. (B) The subcutaneous tumors were harvested and weighted. (C) IHC staining showed that Ki67 expression in AGS cells transfected with si-circCSNKIGI and si-NC. **P $<0.01$.

circCSNK1G1 (Figure 4A). Moreover, it was found that miR-758 mimics transfection remarkably diminished the luciferase activity of circCSNK1G1-WT reporter, but not the activity of circCSNK1G1-Mut in AGS and HGC-27 cells (Figure 4B). RIP assay showed that circCSNK1G1 and miR-758 were significantly enriched in the Ago2 group compared with the IgG group (Figure 4C). Furthermore, miR-758 expression was distinctly highly expressed in normal tissues and cells compared to GC tissues and cells (Figure 4D and E). Besides that, the deletion of circCSNK1G1 upregulated miR-758 expression (Figure 4F). These data showed that circCSNK1G1 could directly interact with miR-758.

\section{CircCSNKIGI Overexpression}

\section{Counteracted miR-758-Mediated Effects on the Progression of GC}

In order to deeply explore the interaction between circCSNK1G1 and miR-758 in GC, the level of miR-758 was assessed in AGS and HGC-27 cells transfected with NC mimics, miR-758 mimics, miR-758 mimics+pcDNA3.1, and miR-758 mimics+pcDNA3.1-circCSNK1G1. RT-qPCR assay indicated that the addition of circCSNK1G1 counteracted the promotive effects of miR-758 mimics on miR-758 expression (Figure 5A). Moreover, the supplementation of
miR-758 hindered the progression of GC, which was neutralized following pcDNA3.1-circCSNK1G1 transfection (Figure 5B-E). The above data indicated that the opposite functions of circCSNK1G1 and miR-758 in GC progression.

\section{ZNF2I7 Was Directly Targeted by miR-758}

According to the prediction of TargetScan, ZNF217 might be a target of miR-758 (Figure 6A). Though GEPIA database, ZNF217 expression was higher expressed in stomach adenocarcinoma (STAD) tissues than that in normal tissues (Figure 6B). To validate the binding ability between miR758 and ZNF217, luciferase reporter and RIP assays were conducted. The results revealed that miR-758 mimics resulted in an obvious reduction in relative luciferase activity of ZNF217-WT but not in ZNF217-Mut (Figure 6C). Meanwhile, miR-758 and ZNF217 were both enriched in the Ago2 group (Figure 6D). Moreover, RT-qPCR and Western blotting revealed that the upregulation of miR-758 decreased ZNF217 expression, and the downregulation of miR-758 increased ZNF217 expression (Figure 6E and F). After that, an increased ZNF217 expression was observed in GC tissues and cells (Figure 6G and H). To summarize, miR-758 negatively regulated ZNF217 level by directly targeting 3'UTR of ZNF217 mRNA. 
A

Target: $5^{\prime}$ aaaccCUGGCACUGGUCACAAa $3^{\prime}$

I।| | ||ा।|ा।

mIRNA : $3^{\prime}$ cCaAucACCUGGUCCAGUGUUU $5^{\prime}$
B

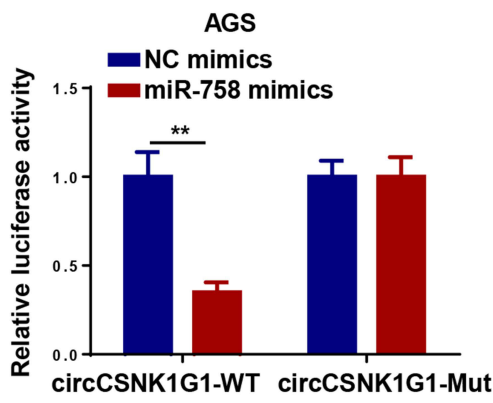

HGC-27

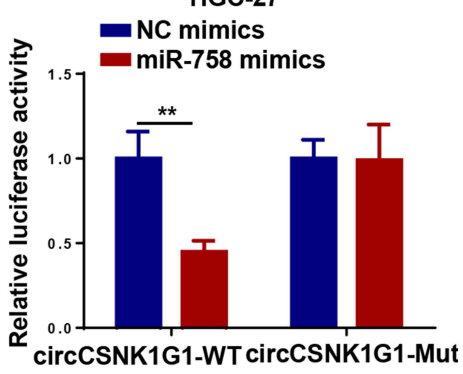

C AGS HGC-27

D
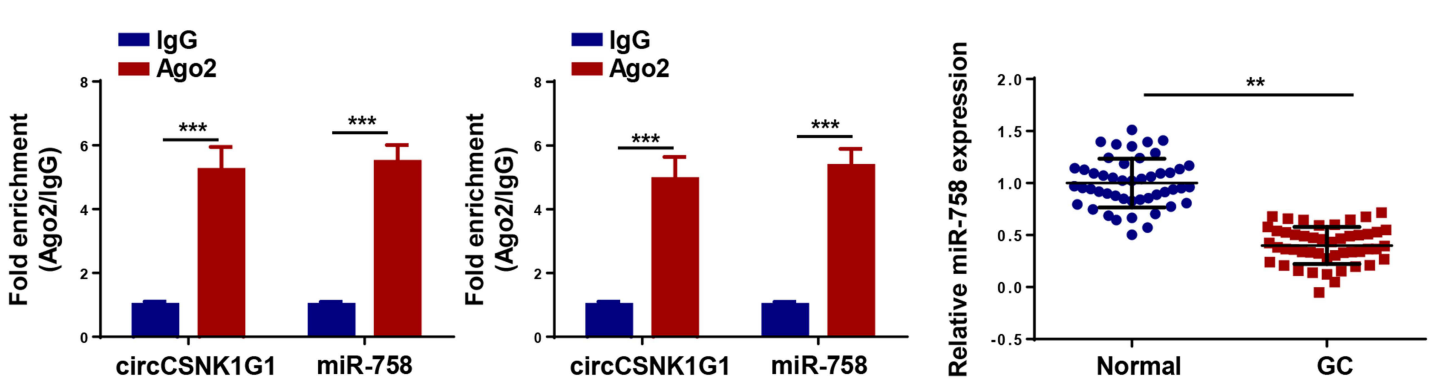

E

F
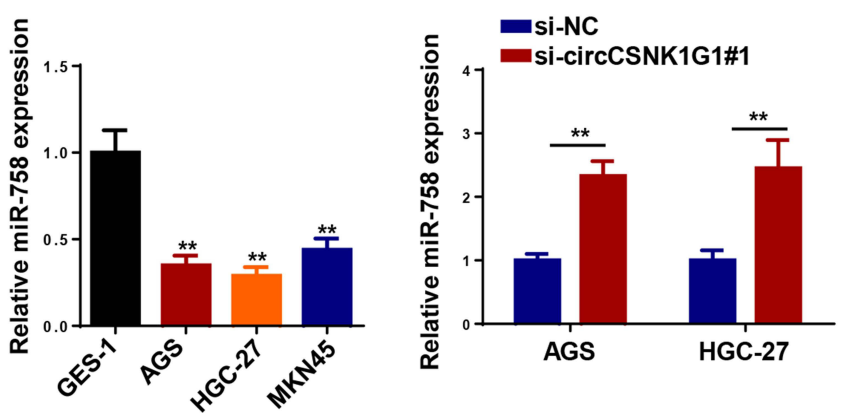

Figure 4 CircCSNKIGI was a sponge of miR-758. (A) Putative binding regions of circCSNKIGI in miR-758 predicted with biological software. (B) Luciferase activity of wild-type or mutant circCSNKIGI in AGS and HGC-27 cells following NC mimics, or miR-758 mimics transfection determined by luciferase reporter assay. (C) Correlations between circCSNKIGI and miR-758 detected by RIP assay. (D) The level of miR-758 in GC tissues and paired normal tissues was measured using RTqPCR. (E) miR-758 in GC cell lines and normal gastric epithelial cell line was detected using RT-qPCR. (F) The expression of miR-758 was detected by RT-qPCR in AGS and HGC-27 cells transfected with si-circCSNKIGI and si-NC. **P $<0.01$ and ***P $<0.001$.

\section{CircCSNKIGI Regulated ZNF2I7 by Sponging miR-758 in GC Cells}

Subsequently, we examined whether ZNF217 was a downstream regulator of circCSNK1G1/miR-758 axis. RTqPCR and Western blotting indicated that ZNF217 was notably downregulated by miR-758 mimics transfection, which could be abolished by pcDNA3.1-ZNF217 in AGS cells (Figure 7A and B). Moreover, the effect of miR-758 mimics on proliferation, migration, invasion, and apoptosis of AGS cells was abolished by upregulating ZNF217 expression (Figure 7C-F). Furthermore, we investigated whether circCSNK1G1 could regulate ZNF217 expression via miR-
758, and the results indicated that interference of circCSNK1G1 downregulated the expression of ZNF217, which was abolished following miR-758 inhibitor transfection (Figure $7 \mathrm{G}$ and $\mathrm{H}$ ). To sum up, these results demonstrated that circCSNK1G1 regulated GC progression via miR-758/ ZNF217 axis.

\section{Discussion}

With the continuous development of next-generation sequencing technology, increasing circRNAs have been identified, as well as their function in human cancers, including GC. For instance, circ-HuR was downregulated 
A

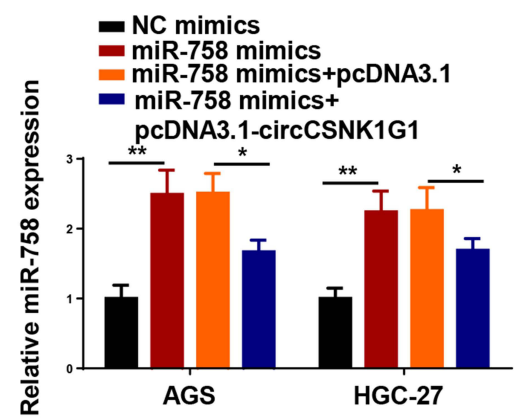

B
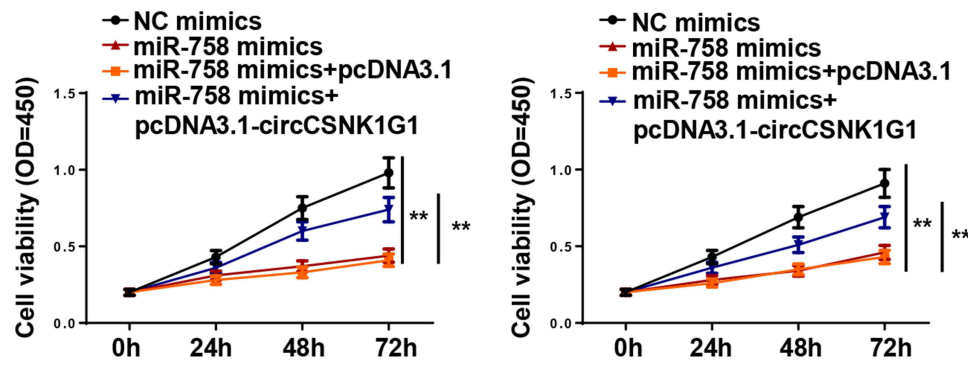

C

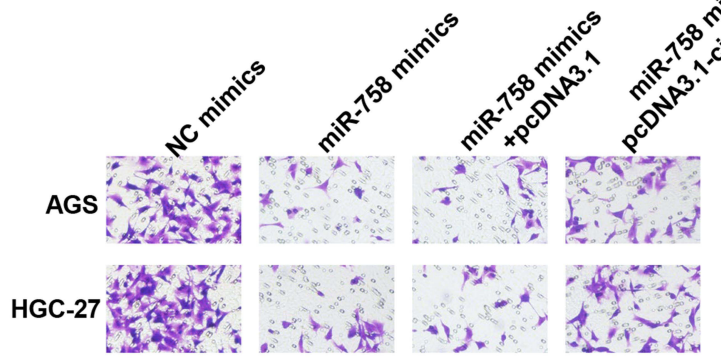

D

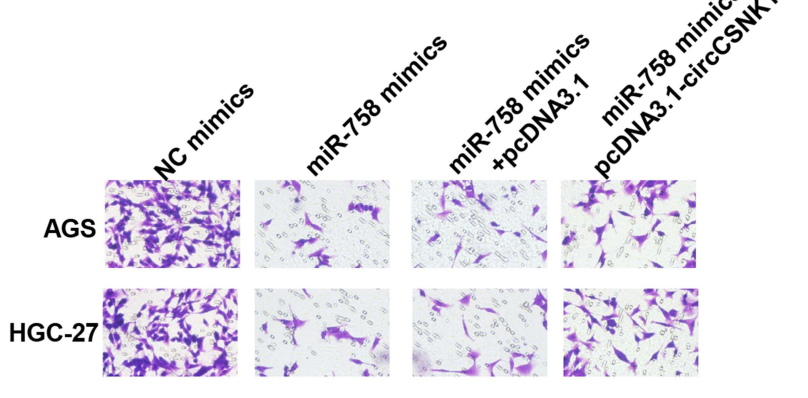

E
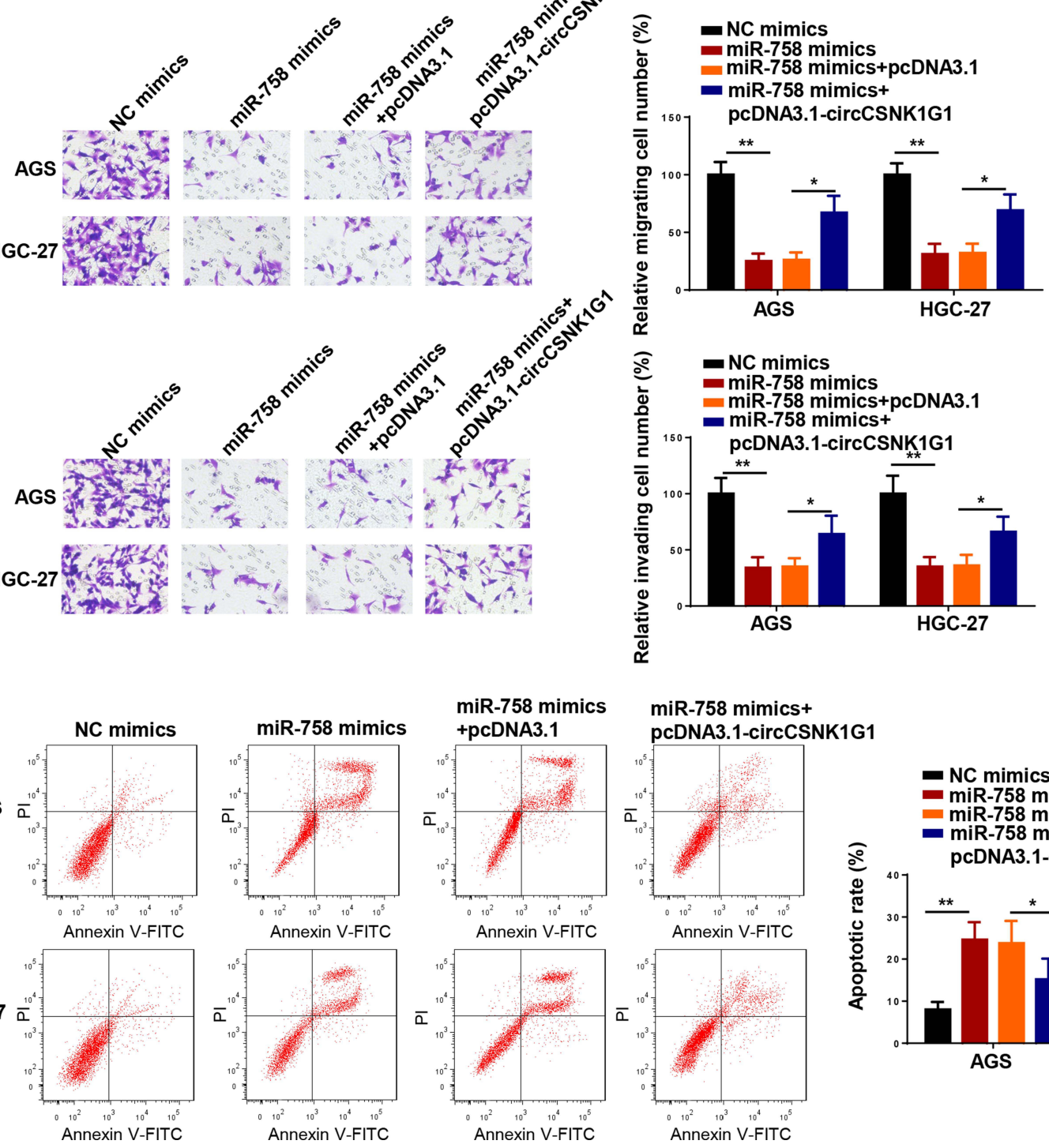

HGC-27
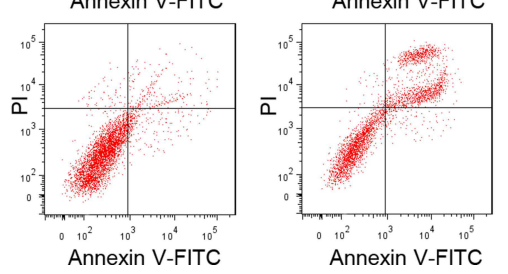

Figure 5 CircCSNKIGI overexpression counteracted miR-758-mediated effects on the progression of GC. (A) The expression of miR-758 was measured by RT-qPCR in AGS and HGC-27 cells transfected with NC mimics, miR-758 mimics, miR-758 mimics+pcDNA3.I, and miR-758 mimics+pcDNA3.I-circCSNKI GI. (B) The proliferation of AGS and HGC-27 cells was measured by CCK-8 assay. (C and D) Transwell was used to detect the migration and invasion of AGS and HGC-27 cells transfected with NC mimics, miR-758 mimics, miR-758 mimics+pcDNA3.I, and miR-758 mimics+pcDNA3.I-circCSNKI GI. (E) Flow cytometry assay showed the apoptosis of AGS and HGC-27 cells transfected with NC mimics, miR-758 mimics, miR-758 mimics+pcDNA3.I, and miR-758 mimics+pcDNA3.I-circCSNKIGI. *P $<0.05$ and $* * P<0.01$. 
A
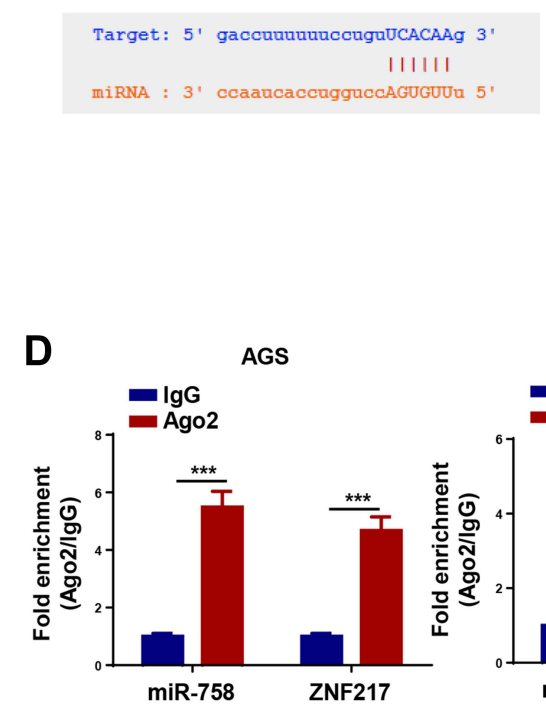

\section{G}

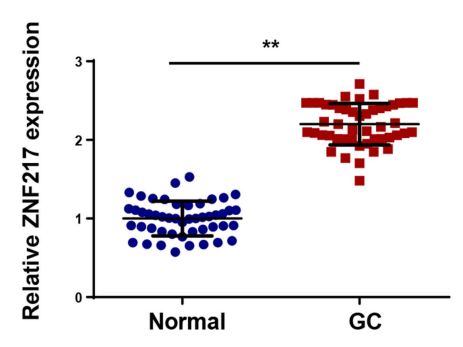

B

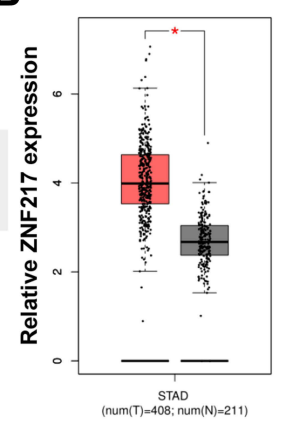

C
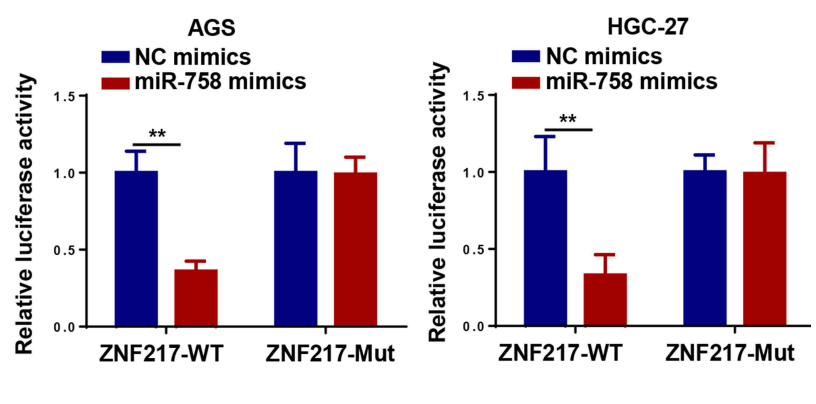

$\mathbf{F}$

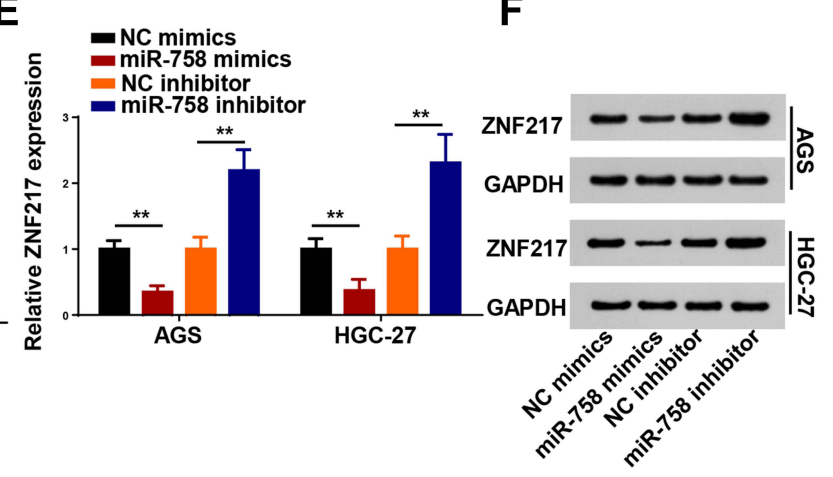

H

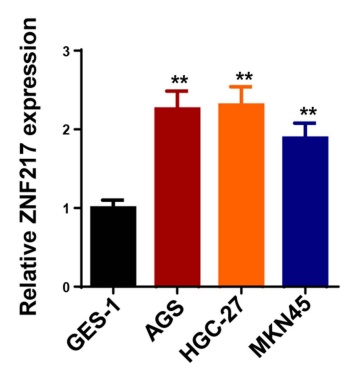

Figure 6 ZNF2 17 was directly targeted by miR-758. (A) Putative binding regions of miR-758 in ZNF2I7 3'UTR. (B) GEPIA database showed ZNF2 I7 expression in stomach adenocarcinoma (STAD) tissues compared with that in normal tissues. (C) Luciferase activity of wild-type or mutant ZNF217 in AGS and HGC-27 cells following NC mimics, or miR-758 mimics transfection determined by luciferase reporter assay. (D) Correlations between ZNF217 and miR-758 detected by RIP assay. (E and F) The expression of ZNF217 was measured by RT-qPCR and Western blotting in AGS and HGC-27 cells transfected with miR-758 mimics or miR-758 inhibitor. (G and $\mathbf{H}$ ) The expression of ZNF2I7 in GC tissues and cells detected by RT-qPCR. **P $<0.01$ and ***P $<0.001$.

in $\mathrm{GC}$, and overexpression of circ-HuR inhibited the growth and metastasis of GC cells. ${ }^{24}$ circRNA_001569 increased proliferation and decreased apoptosis of GC cells via inhibiting miR-145 expression. ${ }^{25}$ CircCSNK1G1, a novel circRNA, was confirmed to be involved in the development of tumors. For example, Yao et al revealed that circCSNK1G1 facilitated hepatocellular carcinoma development by upregulating TRAF6 and MAPK11. ${ }^{26}$ Huang et al reported that circCSNK1G1 accelerated colorectal cancer progression by elevating MYO6 level via interaction with miR-455-3p. ${ }^{27}$ In this research, we focused on the role of circCSNK1G1 in GC. The results showed that circCSNK1G1 was upregulated in GC and deletion of circCSNK1G1 restrained GC progression in vitro and in vivo.
Previous researches have indicated that circRNAs could act as a competing endogenous RNA (ceRNA) of miRNA to exert its effect in cellular physiology. ${ }^{28}$ For example, circCACTIN served as a ceRNA of miR-331 to regulate TGFBR1 level and accelerated GC development. ${ }^{29}$ CircMAT2B facilitated glycolysis and growth of GC through increasing HIF-1 $\alpha$ expression by restraining miR-515-5p. ${ }^{30}$ CircLDLRAD3 promoted GC progression and could function as ceRNA to target NRP2 by sponging miR-224. ${ }^{31}$ In our study, miR-758 was identified as a downstream gene of circCSNK1G1. miR-758 has been verified as a downstream regulator of circRNAs in the tumorigenesis of human cancers. For instance, circRNA RBM33 contributed to the occurrence and development of cervical cancer by sponging miR-758. ${ }^{32}$ 
A
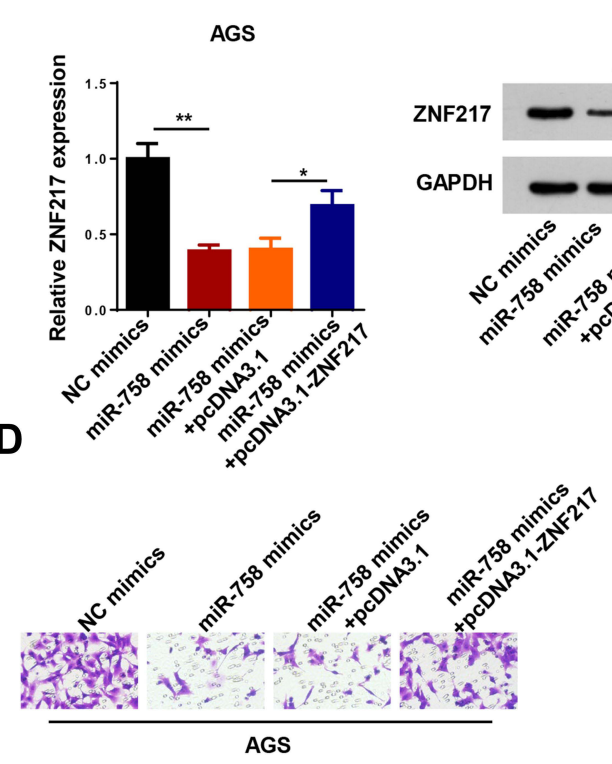

E

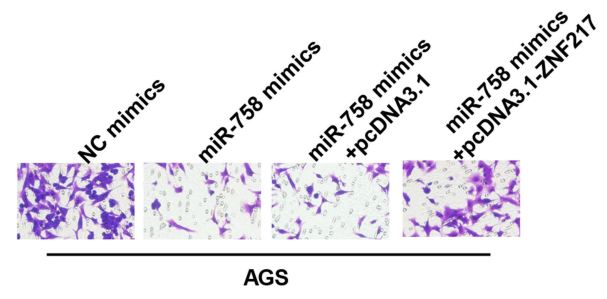

F

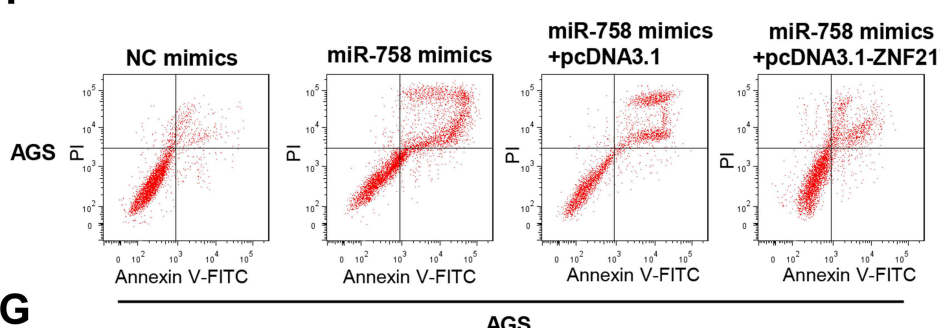

G
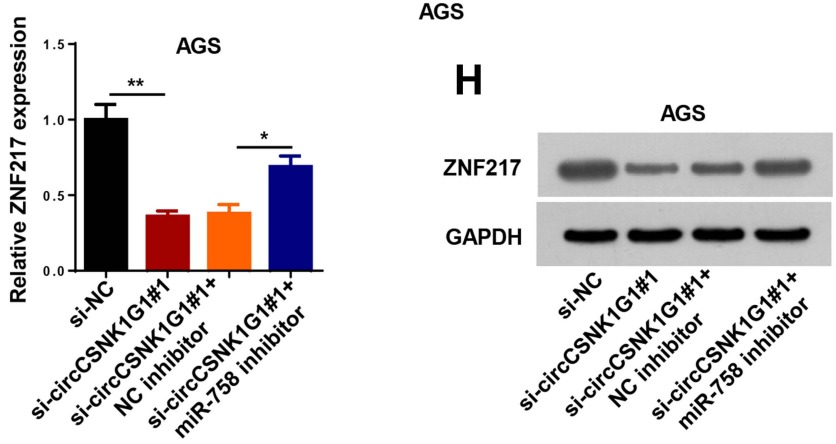

AGS
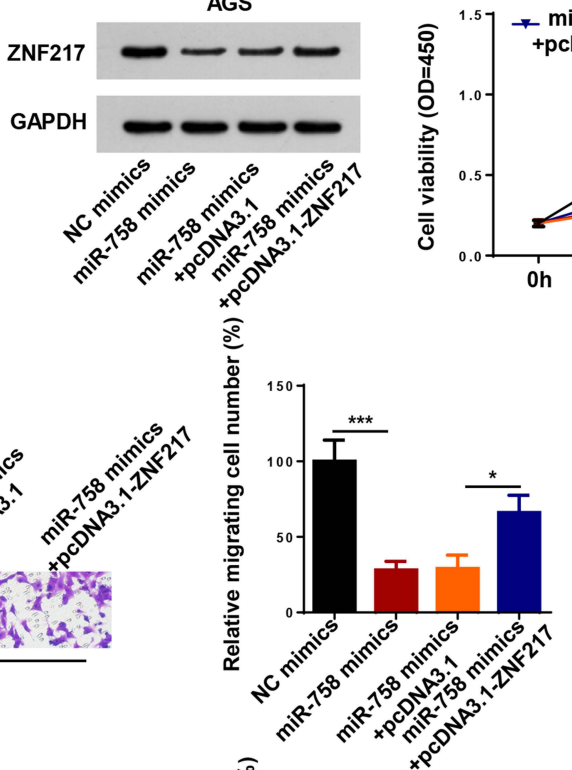

ఏ
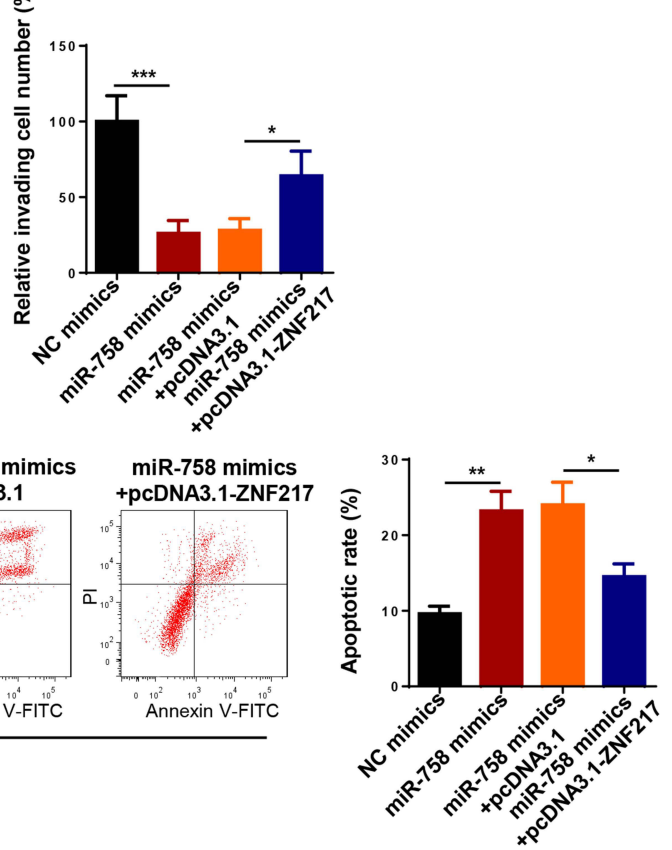

H

Figure 7 CircCSNKIGI regulated ZNF217 by sponging miR-758 in GC cells. (A and B) The expression of ZNF2 I7 was measured by RT-qPCR and Western blotting in AGS cells transfected with NC mimics, miR-758 mimics, miR-758 mimics+pcDNA3.I, and miR-758 mimics+pcDNA3.I-ZNF217. (C-F) CCK-8, Transwell and flow cytometry assays were used to detect the proliferation, migration and invasion and apoptosis of AGS cells transfected with NC mimics, miR-758 mimics, miR-758 mimics+pcDNA3.I, and miR-758 mimics+pcDNA3.I-ZNF217. (G and $\mathbf{H}$ ) The expression of ZNF217 was measured by RT-qPCR and Western blotting in AGS cells transfected with si-NC, sicircCSNKIGI\#I, si-circCSNKIGI\#I+NC inhibitor, and si-circCSNKIGI+miR-758 inhibitor. $* \mathrm{P}<0.05, * * \mathrm{P}<0.01$ and $* * * \mathrm{P}<0.001$. 
Hsa_circ_0002483 facilitates acute myeloid leukemia progression by downregulating miR-758 expression. ${ }^{33}$ Here, we observed that supplementation of miR-758 restrained cell proliferation, migration and invasion, and promoted the apoptosis of GC cells, while circCSNK1G1 overexpression neutralized the effects on GC progression induced by $\mathrm{miR}-758$.

ZNF217 is a potential oncogene located at chromosome $20 \mathrm{q} 13.2$, a region that is frequently involved in tumorigenesis of many cancers. ${ }^{34}$ For example, the upregulation of ZNF217 predicted poor prognosis in breast cancer and promoted breast cancer progression. $^{35}$ LncRNA CTBP1-AS2 enhanced cervical cancer cell proliferation and migration via upregulation of ZNF217. ${ }^{36}$ Moreover, Shida et al indicated high expression of ZNF217 contributed a worse relapse-free survival and overall survival of patients with GC. ${ }^{37}$ In this study, we confirmed that ZNF217 was a target gene of miR-758. Functional assays indicated that the suppressive effect of miR-758 overexpression on GC progression was counteracted by ZNF217 overexpression. In addition, the absence of circCSNK1G1 resulted in a downregulation ZNF217, and miR-758 inhibition abolished the repressive effect of circCSNK1G1 inhibition on ZNF217 expression.

In summary, we demonstrated that circCSNK1G1 stimulated the progression of $\mathrm{GC}$ via regulating the miR-758/ ZNF217 axis. These findings indicated that circCSNK1G1 might be a potential biomarker for GC diagnosis and therapy.

\section{Disclosure}

The authors report no conflicts of interest in this work.

\section{References}

1. Breugom AJ, Swets M, Bosset J-F, et al. Adjuvant chemotherapy after preoperative (chemo)radiotherapy and surgery for patients with rectal cancer: a systematic review and meta-analysis of individual patient data. Lancet Oncol. 2015;16(2):200-207. doi:10.1016/S1470-2045(14) 71199-4

2. Zhao L, Jiang L, He L, et al. Identification of a novel cell cycle-related gene signature predicting survival in patients with gastric cancer J Cell Physiol. 2019;234(5):6350-6360. doi:10.1002/jcp.27365

3. Chen W, Zheng R, Baade PD, et al. Cancer statistics in China, 2015. CA Cancer J Clin. 2016;66(2):115-132. doi:10.3322/caac.21338

4. Rahman R, Asombang AW, Ibdah JA. Characteristics of gastric cancer in Asia. World J Gastroenterol. 2014;20(16):4483-4490. doi:10.3748/ wjg.v20.i16.4483

5. Lasithiotakis K, Antoniou SA, Antoniou GA, et al. Gastrectomy for stage IV gastric cancer. a systematic review and meta-analysis. Anticancer Res. 2014;34(5):2079-2085.

6. Li Z, Ruan Y, Zhang H, et al. Tumor-suppressive circular RNAs: mechanisms underlying their suppression of tumor occurrence and use as therapeutic targets. Cancer Sci. 2019;110(12):3630-3638. doi:10.1111/cas.14211
7. Guo JU, Agarwal V, Guo H, et al. Expanded identification and characterization of mammalian circular RNAs. Genome Biol. 2014;15(7):409. doi:10.1186/s13059-014-0409-z

8. Barrett SP, Wang PL, Salzman J. Circular RNA biogenesis can proceed through an exon-containing lariat precursor. eLife. 2015;4: e07540. doi:10.7554/eLife.07540

9. Patop IL, Kadener S. circRNAs in Cancer. Curr Opin Genet Dev. 2018;48:121-127. doi:10.1016/j.gde.2017.11.007

10. Liu Z, Yu Y, Huang Z, et al. CircRNA-5692 inhibits the progression of hepatocellular carcinoma by sponging miR-328-5p to enhance DAB2IP expression. Cell Death Dis. 2019;10(12):900. doi:10.1038/ s41419-019-2089-9

11. Zeng K, Chen X, Xu M, et al. CircHIPK3 promotes colorectal cancer growth and metastasis by sponging miR-7. Cell Death Dis. 2018;9 (4):417. doi:10.1038/s41419-018-0454-8

12. Yu X, Ding H, Yang L, et al. Reduced expression of circRNA hsa_circ_0067582 in human gastric cancer and its potential diagnostic values. J Clin Lab Anal. 2020;34(3):e23080. doi:10.1002/jcla.23080

13. Sun B, Sun H, Wang Q, et al. Circular RNA circMAN2B2 promotes growth and migration of gastric cancer cells by down-regulation of miR-145. J Clin Lab Anal. 2020;34(6):e23215. doi:10.1002/jcla.23215

14. Zhang Y, Xia L, Wu J, et al. Hsa_circ_0023642 promotes proliferation, invasion, and migration of gastric cancer by sponging microRNA-223. J Clin Lab Anal. 2020;34(10):e23428. doi:10.1002/ jcla. 23428

15. Ding HX, Xu Q, Wang B-G, et al. MetaDE-based analysis of circRNA expression profiles involved in gastric cancer. Dig Dis Sci. 2020;65(10):2884-2895. doi:10.1007/s10620-019-06014-6

16. You Y, Que K, Zhou Y, et al. MicroRNA-766-3p inhibits tumour progression by targeting Wnt3a in hepatocellular carcinoma. Mol Cells. 2018;41(9):830-841. doi:10.14348/molcells.2018.0181

17. Wang Q, Yu J. MiR-129-5p suppresses gastric cancer cell invasion and proliferation by inhibiting COL1A1. Biochem Cell Biol. 2018;96 (1):19-25. doi:10.1139/bcb-2016-0254

18. $\mathrm{Hu} \mathrm{X}$, Zhang M, Miao J, et al. miRNA-4317 suppresses human gastric cancer cell proliferation by targeting ZNF322. Cell Biol Int. 2018;42(8):923-930. doi:10.1002/cbin.10870

19. Liao Z, Li Y, Zhou Y, et al. MicroRNA-197 inhibits gastric cancer progression by directly targeting metadherin. Mol Med Rep. 2018;17 (1):602-611. doi:10.3892/mmr.2017.7908

20. Wu X, Chen B, Shi H, et al. miR-758-3p suppresses human bladder cancer cell proliferation, migration and invasion by targeting NOTCH2. Exp Ther Med. 2019;17(5):4273-4278. doi:10.3892/ etm.2019.7400

21. Hu X, Li Y, Kong D, et al. Long noncoding RNA CASC9 promotes LIN7A expression via miR-758-3p to facilitate the malignancy of ovarian cancer. $J$ Cell Physiol. 2019;234(7):10800-10808. doi:10.1002/jcp. 27903

22. Song T, Hou X, Lin B. MicroRNA-758 inhibits cervical cancer cell proliferation and metastasis by targeting HMGB3 through the WNT/ beta-catenin signaling pathway. Oncol Lett. 2019;18(2):1786-1792. doi:10.3892/ol.2019.10470

23. Guo J, Zhang Z, Pan L, et al. Identification of miR-758-3p as potential modulator of CBX5 expression in gastric cancer. Technol Cancer Res Treat. 2018;17:1533033818816061. doi:10.1177/ 1533033818816061

24. Yang F, Hu A, Li D, et al. Circ-HuR suppresses HuR expression and gastric cancer progression by inhibiting CNBP transactivation. Mol Cancer. 2019;18(1):158. doi:10.1186/s12943-019-1094-Z

25. Shen F, Liu P, Xu Z, et al. CircRNA_001569 promotes cell proliferation through absorbing miR-145 in gastric cancer. $J$ Biochem. 2019;165(1):27-36. doi:10.1093/jb/mvy079

26. Yao Z, Xu R, Yuan L, et al. Circ_0001955 facilitates hepatocellular carcinoma (HCC) tumorigenesis by sponging miR-516a-5p to release TRAF6 and MAPK11. Cell Death Dis. 2019;10(12):945. doi:10.1038/s41419-019-2176-y 
27. Huang $X$, Shen $X$, Peng L, et al. CircCSNK1G1 contributes to the development of colorectal cancer by increasing the expression of MYO6 via competitively targeting miR-455-3p. Cancer Manag Res. 2020;12:9563-9575. doi:10.2147/CMAR.S262007

28. Qi X, Zhang D-H, Wu N, et al. ceRNA in cancer: possible functions and clinical implications. $J$ Med Genet. 2015;52(10):710-718. doi:10.1136/jmedgenet-2015-103334

29. Zhang L, Song X, Chen X, et al. Circular RNA circCACTIN promotes gastric cancer progression by sponging miR-331-3p and regulating TGFBR1 expression. Int J Biol Sci. 2019;15(5):1091-1103. doi:10.7150/ijbs.31533

30. Liu J, Liu H, Zeng Q, et al. Circular RNA circ-MAT2B facilitates glycolysis and growth of gastric cancer through regulating the miR-515-5p/HIF-1alpha axis. Cancer Cell Int. 2020;20:171. doi:10.1186/s12935-020-01256-1

31. Wang Y, Yin H, Chen X. Circ-LDLRAD3 enhances cell growth, migration, and invasion and inhibits apoptosis by regulating MiR-224-5p/NRP2 axis in gastric cancer. Dig Dis Sci. 2021. doi:10.1007/s10620-020-06733-1

32. Ding Y, Yuan X, Gu W. Circular RNA RBM33 contributes to cervical cancer progression via modulation of the miR-758-3p/PUM2 axis. J Mol Histol. 2021;52:173-185. doi:10.1007/s10735-020-09933-1
33. Xiao Y, Ming X, Wu J. Hsa circ 0002483 regulates miR-758-3p/ MYC axis to promote acute myeloid leukemia progression. Hematol Oncol. 2020;39:243-253. doi:10.1002/hon.2829

34. Kallioniemi A, Kallioniemi OP, Piper J, et al. Detection and mapping of amplified DNA sequences in breast cancer by comparative genomic hybridization. Proc Natl Acad Sci U S A. 1994;91(6):2156-2160. doi:10.1073/pnas.91.6.2156

35. Vendrell JA, Thollet A, Nguyen NT, et al. ZNF217 is a marker of poor prognosis in breast cancer that drives epithelial-mesenchymal transition and invasion. Cancer Res. 2012;72(14):3593-3606. doi:10.1158/0008-5472.CAN-11-3095

36. Yang S, Shi F, Du Y, et al. Long non-coding RNA CTBP1-AS2 enhances cervical cancer progression via up-regulation of ZNF217 through sponging miR-3163. Cancer Cell Int. 2020;20:343. doi:10.1186/s12935-020-01430-5

37. Shida A, Fujioka S, Kurihara $\mathrm{H}$, et al. Prognostic significance of ZNF217 expression in gastric carcinoma. Anticancer Res. 2014;34 (9):4813-4817.

\section{Publish your work in this journal}

Cancer Management and Research is an international, peer-reviewed open access journal focusing on cancer research and the optimal use of preventative and integrated treatment interventions to achieve improved outcomes, enhanced survival and quality of life for the cancer patient.
The manuscript management system is completely online and includes a very quick and fair peer-review system, which is all easy to use. Visit http://www.dovepress.com/testimonials.php to read real quotes from published authors. 